\title{
Aprendizaje basado en proyectos mediados por Tic para desarrollar competencias matemáticas en estudiantes de secundaria
}

\author{
Mg. Gloria Marilú Hilario Bacilio \\ ghilariob@ucvvirtual.edu.pe \\ 0000-0001-5148-7806 \\ Universidad César Vallejo \\ Trujillo - Perú
}

\section{RESUMEN}

El presente estudio tuvo como finalidad determinar la influencia del aprendizaje basado en proyectos mediados por TIC en el desarrollo de competencias matemáticas de estudiantes del quinto grado nivel secundario de la institución educativa José Antonio Encinas de Trujillo. Para tal fin, se realizó una investigación tipo aplicada, con enfoque cuantitativo y diseño cuasi experimental. La población estuvo conformada por 57 estudiantes del VII ciclo quinto grado de la institución educativa José Antonio Encinas, dividido en dos grupos; grupo control con 27 estudiantes (5to "A") y uno grupo experimental con 30 estudiantes (5to "B"). La técnica para el recojo de datos fue la encuesta y el instrumento una prueba escrita. Constó de 16 ítems, validado por cinco jueces expertos y con un análisis de fiabilidad obtenido con el test KR 20=0,8079. Se aplicó mediante formulario Google al inicio del estudio a ambos grupos y después de 22 sesiones, tiempo que duró el programa, se aplicó al grupo experimental. Se calculó la prueba de hipótesis usando el test de U de Mann Whitney obteniéndose p: valor $0,00<0,01$ en competencias matemáticas y dimensiones, concluyendo que el aprendizaje basado en proyectos mediados por Tic influye positiva y significativamente en las competencias matemáticas.

Palabras claves: método de proyectos; competencias matemáticas; TIC. 


\title{
ICT-mediated project-based learning to develop mathematical competencies in high school students
}

\begin{abstract}
The purpose of this study was to determine the influence of project-based learning mediated by ICT in the development of mathematical competencies of students of the fifth-grade secondary level of the educational institution José Antonio Encinas de Trujillo. For this purpose, an applied type research was carried out, with a quantitative approach and a quasi-experimental design. The population was made up of 57 students of the VII cycle of fifth grade of the educational institution José Antonio Encinas, divided into two groups; control group with 27 students (5th "A") and one experimental group with 30 students (5th "B"). The technique for data collection was the survey and the instrument a written test. It consisted of 16 items, validated by five expert judges and with a reliability analysis obtained with the KR $20=0.8079$ test. It was applied by Google form at the beginning of the study to both groups and after 22 sessions, the duration of the program, it was applied to the experimental group. The hypothesis test was calculated using the Mann Whitney $U$ test, obtaining p: value $0.00<0.01$ in mathematical competencies and dimensions, concluding that project-based learning mediated by Tic positively and significantly influences mathematical competencies.
\end{abstract}

Keywords: project method; mathematical skills; Tic.

Artículo recibido: 18. Junio. 2021 Aceptado para publicación: 26. Julio. 2021 Correspondencia: ghilariob@ucvvirtual.edu.pe Conflictos de Interés: Ninguna que declarar 


\section{INTRODUCCIÓN}

La matemática ha sido siempre una asignatura que ha demandado un mayor esfuerzo en su aprendizaje en comparación a otras. Soler et al. (2013) sostienen que los factores que afectan su aprendizaje están relacionados con la estructura curricular, cantidad de estudiantes por aula, metodología del docente. Mizala y Romaguera (1999, como se citó en Huanca y Moreno, 2019) afirman que las variables que intervienen están asociadas tanto a variables familiares como las relacionadas con la escuela como clima institucional, infraestructura, metodología y recursos pedagógicos. Así mismo, Dávila (2011, como se citó en Wampash, 2018) sostiene que su aprendizaje guarda relación con la motivación intrínseca, ambiente físico, metodología, estilos y ritmos de cada sujeto que aprende.

Teniendo en cuenta estos componentes y tomando los aportes de las teorías sicológicas y pedagógicas que proponen metodologías activas; actualmente diversos países están modificando sus estructuras curriculares, apostando por estrategias de enseñanza que ayuden a desarrollar competencias, como el método basado en problemas, estudio de casos, gamificación, el método basado en proyectos, haciendo uso de herramientas multimedia y TIC.

Como se sabe, la matemática es un conjunto de lenguajes formales, que para su internalización se requiere de un razonamiento lógico, porque relaciona un conjunto de conceptos abstractos como sistemas numéricos, equivalencias, regularidades, formas, transformaciones, inferencias y probabilidades; entonces, si su enseñanza sigue un enfoque algorítmico, la Matemática se convierte en una ciencia de fórmulas y procedimientos rígidos, fría, nada interesante, nada significativa, poco útil y difícil de comprender.

Corbalán (1995) habla de una Matemática aplicada a la vida cotidiana y manifiesta que, ante una misma realidad, cada estudiante lo observa de acuerdo a sus propios intereses, expectativas y como lo enseñaron a percibirla. En este sentido, la labor del docente es guiar a los aprendices a observarla en su vida diaria, que no lo vean como una algo inconexo a su realidad, sino que encuentren patrones y relaciones que lo vinculen con ella. Para esto, el docente se debe valer de metodologías integradoras, que los motive aprender, que le permita construir su conocimiento; actuando en contextos reales, siendo él protagonista activo, presente y gestor de acciones que generen aprendizajes, teniendo 
a su favor el uso de la tecnología, la cual ayudará a mirar la situación de estudio en diversos contextos.

Los resultados de las pruebas estandarizadas internacionales y nacionales, muestran lo que están aprendiendo nuestros estudiantes en el área de Matemática. El Minedu, a través de la UMC, mostró los puntajes de la última evaluación internacional PISA 2018, donde nuestros estudiantes obtuvieron un promedio de 400 puntos, muy por debajo del nivel 2 (420 y 482), ubicándolos en nivel deficiente y caracterizándolos con escaso razonamiento lógico para resolver situaciones en diversos contextos (Oficina de medición de la calidad de los aprendizajes [UMC], 2018). Así mismo, en la evaluación estandarizada nacional (ECE) 2019, el 32,1\% de los participantes se encuentran en escala previo al inicio; el $33,0 \%$ en inicio, el 17,3\% en proceso y solo en satisfactorio 17,7\%. (Ministerio de educación: Sistema de consulta de resultados de evaluaciones [MINEDU-SICRECE], 2019 , p.4). En conclusión, más de la mitad $(65,1 \%)$ se encuentran en nivel de logro previo al inicio e inicio, calificándolos como estudiantes que no lograron aprendizajes necesarios para su nivel, resolviendo solo situaciones problemáticas en contextos cercanos y aplicando procedimientos muy elementales con respecto al ciclo evaluado (MINEDUSICRECE, 2018, p.8).

Por lo tanto, es necesario buscar nuevas formas de enseñar y aprender. La presente investigación, propone al "Aprendizaje basado en proyectos" como estrategia que permita disminuir los porcentajes de la escala inicio y previo al inicio en estas competencias.

Este método, está considerado en el grupo de metodologías activas, que, por su estructura y finalidad, permite actuar con un proyecto en una realidad concreta, involucrando a los sujetos que aprenden, permitiéndoles ser actores directos de la solución, logrando incrementar su motivación por aprender.

Además, es eficaz dentro de un enfoque por competencias. García (2018) sostiene, que las actividades mediante proyectos permiten desarrollar y mantener la motivación, ayuda a lograr un aprendizaje significativo y por ende duradero, estimula su pensamiento crítico, reflexivo, creativo; ayuda a integrar conocimientos mediante la interdisciplinariedad y el trabajo colaborativo. (p. 33-35). Así mismo, Rodríguez (2018), lo cataloga como una estrategia que ayuda a organizar el pensamiento y juicio crítico, permite elaborar hipótesis y buscar información relevante, mezclando lo que descubre con lo que sabe (p.9). También, permite involucrarse en situaciones complejas haciéndolo adquirir nuevos 
saberes que le servirán para solucionar una situación mediante las fases de un proyecto (Gutiérrez et al. 2013, como se citó en Castro, 2020, p. 7).

Las características del aprendizaje basado en proyectos son suficientes para el desarrollo de competencias matemáticas y si le sumamos el uso de las TICS, se convierte en una estrategia poderosa para el docente y estudiante orientado al lograr el perfil de egreso de todo sistema educativo.

Por consiguiente, este estudio busca determinar un marco conceptual que explique que el aprendizaje basado en proyectos utilizando las Tic, pueda verse como una estrategia de enseñar y aprender, de trabajo colaborativo, donde estudiantes y docentes se involucran en el estudio de situaciones reales teniendo como evidencia un producto tangible o intangible que será la propuesta de solución de dicha problemática.

Bajo esta perspectiva, la pregunta a responder en la presente investigación fue: ¿De qué manera el aprendizaje basado en proyectos mediados por TIC influirá en el desarrollo de las competencias matemáticas de los estudiantes del quinto grado del nivel secundario de la Institución Educativa José Antonio Encinas de Trujillo?”, también se trazó cumplir con el objetivo: "Determinar de qué manera el aprendizaje basado en proyectos mediados por TIC, influirá en el desarrollo de las competencias matemáticas de los estudiantes del quinto grado del nivel secundario de la institución educativa José Antonio Encinas de Trujillo. La hipótesis sustantiva del presente estudio parte del supuesto que una intervención pedagógica basada en la metodología del aprendizaje basado en proyectos mediados por TIC, influirá significativamente en el desarrollo de las competencias matemáticas de los estudiantes del quinto grado del nivel secundario de la institución educativa José Antonio Encinas de Trujillo.

Entre los trabajos que guardan relación con la presente investigación tenemos a Flores y Juárez (2017), quienes estudiaron al aprendizaje basado en proyectos en la mejora de la motivación hacia las matemáticas y hacia las competencias matemáticas relacionadas con la Geometría y Trigonometría a través de un proyecto contextualizado. La investigación fue un estudio de caso. cuyo producto final fue la elaboración de una maqueta como propuesta de solución, concluyendo que los estudiantes con la aplicación del método de proyectos, mostraron aprendizajes de orden superior, mejoraron su trabajo colaborativo, su pensamiento crítico y creativo, así como mejoró su motivación con respecto a las matemáticas. 
Barrera (2017) estudió el Impacto del Aprendizaje basado en proyectos colaborativos ABPC, mediados por TIC en el desarrollo de competencias en estadística. La investigación fue de tipo explicativa, con diseño cuasi experimental. Durante todo el periodo los estudiantes elaboraron cuatro proyectos. Los resultados mostraron una mejora de las competencias en estadística. Además, la inclusión de TIC permitió aprender nuevas herramientas informáticas, favoreciendo su formación académica.

Vargas et al. (2020), su estudio tuvo como finalidad, determinar si el aprendizaje basado en proyectos mediados por las TICS, favorece el aprendizaje de las operaciones básicas matemáticas. La investigación fue de tipo mixta (Cuantitativo - cualitativo), con un diseño cuasi experimental, obteniéndose que el ABP mejora los aprendizajes de la matemática, además mejoró su autorregulación y autocontrol con respecto a sus actitudes positivas ante la matemática.

El Aprendizaje basado en proyectos, se enmarca dentro del enfoque de las corrientes sicopedagógicas constructivistas como la teoría socio cultural de Lev Semiónovich Vygotsky, quien sostiene que los sujetos que aprenden se interrelacionan con su contexto, entre sus pares y su docente, actuando éste como el que diseña estrategias interactivas que promuevan zonas de desarrollo próximo (Chaves, 2001, p.63). La teoría Socio cultural, también afirma que el aprendizaje o funciones mentales superiores se construyen en interrelación con el ambiente social donde se desenvuelve el sujeto aprendiz, así como, a través de situaciones desafiantes guiadas por un experto. También se sustenta en la teoría del aprendizaje significativo de David Ausubel, quien sostiene que el aprendizaje se construye a partir de los saberes previos del sujeto, y se correlaciona con el conocimiento integrado que le proporciona el docente; además se vale de organizadores como mapas mentales, conceptuales, redes semánticas, analogías, resúmenes (Fernández, 2016, como se citó en Arias y Oblitas, 2014, p. 458).

Al hablar de aprendizaje basado en proyectos, no se puede dejar de lado a Jhon Dewey (1899), quien fue uno de los primeros que habló de proyectos, con su idea de trabajos constructivos, y más tarde su discípulo Kilpatrick (1918) lo mejoró en su ensayo titulado "Método de Proyectos" para ser utilizado en el campo educativo. Para Kilpatrick el comportamiento, es la respuesta de un organismo ante una situación, la misma que genera una necesidad, y para satisfacerla se traza una meta. Esta, para ser cumplida sigue un proceso en donde los sujetos se interrelacionan entre sí y experimentan placer al 
cumplirla. Todo este camino, es lo que Kilpatrick llama proyecto. Además, el método de proyectos da continuidad a la educación, a la vida, al saber y al hacer (London, 1997, p.8). El Minedu (2013) define a un proyecto como una forma de planificación integradora, holística e intercultural, cuya finalidad es movilizar un conjunto de capacidades, habilidades y destrezas, se desarrolla en un periodo de tiemplo a través etapas como la planificación, implementación, comunicación y evaluación de actividades (p. 15). Así mismo, De la Puente et al. (2019) un proyecto es la suma de conocimientos, gestión de recursos, auto dirección.

En este sentido, el aprendizaje basado en proyectos, da apertura al desarrollar competencias, porque activa potencialidades, habilidades personales y sociales, conocimientos, destrezas, actitudes en los sujetos que aprenden y los prepara para la vida. Cisneros (1996), sostuvo que el objetivo de esta metodología es lograr un aprendizaje significativo, construido desde una actividad; es decir, que el sujeto aprende preguntando, participando, investigando, trabajando, construyendo; esto implica que el estudiante se sitúe en una realidad, la observe y trate de transformarla para mejorarla y para esto es indispensable la acción (p. 155).

Así mismo, el Instituto tecnológico y de estudios superiores de Monterrey (s.f) sostiene que el aprendizaje mediante proyectos permite a los estudiantes a ser responsables de lo que aprenden, desencadenando en ellos el amor y deseo por aprender, considerándose actores de cambio al aplicar lo que aprendieron en la solución de situaciones problematizadoras (p.3). El mismo instituto también sostiene que este método busca que el aprendiz se involucre con su entorno y aplique conocimientos multidisciplinarios para dar solución a situaciones de su comunidad, esto implica que planifique, implemente y evalúe proyectos (Instituto tecnológico y de estudios superiores de Monterrey ,2010, p.1). En suma, el aprendizaje mediante proyectos, se convierte en una metodología de apoyo para el docente como mediador de la construcción de los aprendizajes, y para el estudiante como constructor y autorregulador de sus propias experiencias, en este sentido cada uno tiene un rol que cumplir.

El docente en esta metodología es guía, tutor y orientador durante todo el proceso de duración del proyecto. Garay (2019) asevera que el maestro debe tener muy claro hacia donde dirige a sus aprendices en búsqueda de los aprendizajes (p.29). Del mismo modo, Bautista (2018) menciona que la función del educador es orientar al aprendiz a tomar 
decisiones lógicas y al mismo tiempo generar actitudes sociales positivas sobre tales decisiones, cuyo producto es la elaboración y sustentación de un proyecto.

El rol que cumple el alumno en esta metodología, es el de un constructor de aprendizajes. No es un ente pasivo, que se remite solo a una escucha activa, por el contrario, es un ente sumamente activo, al desarrollar procesos cognitivos de orden superior, como el pensamiento crítico, complejo, creativo, inductivo, deductivo, analítico sintético, reflexivo y todas sus combinaciones (Pujol, 2017, p. 10).

Barrera (2017), sostiene que el aprendizaje mediante proyectos usado en el proceso de E-A, brinda ventajas al estudiante como: a) Acrecienta la motivación por aprender. b) Hay conexión de la escuela y el contexto real, la teoría y práctica van de la mano. c) Admite el trabajo colaborativo, acrecentando el conocimiento. d) Permite desarrollar habilidades blandas. e) Ofrece la oportunidad de proyección a la comunidad, proponiendo y desarrollando proyectos simples y complejos, de corta, mediano y largo plazo. f) Mejora la autoestima, porque el sujeto se siente reconocido como parte de una solución. g) Auto regula su aprendizaje, siendo consciente de lo que necesita aprender, conocer y desaprender para volver aprender. h) Auto direcciona su aprendizaje. I) Uso de la tecnología, j) Se convierte en autodidacta y descubre nuevos aprendizajes.

El Instituto tecnológico y de estudios superiores de Monterrey (s.f), manifiesta que las ventajas para el docente que incorpora a su labor pedagógica esta metodología le permite: a) Ser más planificador b) Hablar menos. c) Actuar menos como experto d) Ser multidisciplinario. d) Trabajar más en colaboración. e) Usar más variedad de fuentes originales del momento que secundarias h) Realizar más evaluación multidimensional y basada en desempeños h) Realizar menos pruebas a lápiz y papel. j) Realizar menos evaluaciones memorísticas k) Utilizar variedad de materiales, medios y recursos tecnológicos 1) Salir al trabajo de campo (p. 25).

Por otro lado, el Minedu (2013) manifiesta, que el docente al asumir su trabajo pedagógico en un enfoque por competencias, también debe reevaluar su forma de actuar y pensar. En ese sentido, los proyectos de aprendizaje son una alternativa para organizar situaciones didácticas directas al desarrollo de competencias, por lo cual, propone características peculiares, que se debe cumplir al organizar un proyecto, entre ellas: a) El proyecto debe estar dirigido a resolver una situación o problema. b) El estudiante debe ser el protagonista en todo el proceso c) Debe buscarse la multidisciplinariedad. d) En su 
planificación participa el docente y el estudiante y otros agentes educativos e) Se debe trabajar en pequeños grupos f) El proyecto debe terminar con un producto concreto. g) Debe tener una duración. h) estar organizado en etapas (p. 17).

Al respecto, el Minedu considera cuatro fases en un proyecto: planificación, implementación, comunicación y evaluación. En cada etapa los estudiantes investigan a profundidad, realizan trabajos colaborativos, buscan la interdisciplinariedad, presentan sus ideas de manera creativa. Las TIC se convierten en una herramienta fundamental de apoyo, los estudiantes pueden recopilar, ampliar, analizar, recrear, organizar y compartir información de cualquier tipo; probar modelos, hacer presentaciones en diferentes formatos, comunicarse con personas de otras culturas, entre otras.

Para el docente, el uso de esta metodología, también lo motiva y obliga a usar las TICS, pues, su rol es esquematizar experiencias de aprendizaje y guiar a los estudiantes a que lo aprovechen al máximo durante el desarrollo de su proyecto, para esto, no es necesario que sea un ducho en la materia, su fortaleza recae en su conocimiento pedagógico, disciplinar y su capacidad didáctica (Minedu, 2018, p.6). Badia y García (2006, citado en Barrera, 2017) afirman que, en todo proceso de E-A utilizando las Tic, se dan las siguientes interrelaciones: docente-Tic-estudiante, docente-Tic-contenido, estudianteTic-contenido (p. 29), en las cuales se utilizan herramientas web 2.0, entornos virtuales de aprendizaje (EVA), ambientes virtuales de aprendizaje (AVA), objetos virtuales de aprendizaje (OVA) y todas las herramientas posibles, capaces de hacer del aprendizaje una experiencia acogedora, integradora y útil para la vida.

Cuando hablamos de Competencias matemáticas no podemos dejar de lado la teoría del desarrollo cognitivo de Jean Piaget y Collins. El primero, en su teoría sostuvo que el desarrollo cognitivo pasa por niveles: preoperatorio (4-6), Temprano de operaciones concretas (7-9), Medio de operaciones concretas (10-12), último de operaciones concretas (13-15). El segundo, plantea a la matemática como una estructura lógica, coherente, organizada en niveles de gradualidad. Estas dos teorías ayudan al docente a la hora de elegir sus proyectos, programas y hacer de la matemática un sentido concreto y significativo (Collins, 1982, como se citó en Nortes y Martínez, 1994).

Así mismo, George Pólya, con su método de resolución de problemas, aportó significativamente al desarrollo de competencias matemáticas; considerando que para 
resolver un problema se debe pasar por cuatro etapas: Comprender el problema, Concebir un plan, Ejecución del plan, Examinar la solución obtenida (May Cen, I., 2015, p. 2015). Tobón (2012, citado en Cázarez, 2015) define a la competencia como "Actuaciones integrales para identificar, interpretar, argumentar y resolver problemas con idoneidad, metacognición y compromiso ético movilizando los diversos saberes: saber ser, saber hacer y saber conocer" (p.62). Para el Minedu (2017) competencia es "la facultad que tiene una persona de combinar un conjunto de capacidades a fin de lograr un propósito específico en una situación determinada, actuando de manera pertinente y con sentido ético" (p. 192).

Si hablamos de competencia matemática la OCDE (2016b, citado en Minedu-UMC, 2017) sostiene que es la "Capacidad para formular, emplear e interpretar las matemáticas en distintos contextos. Incluye el razonamiento matemático y la utilización de conceptos, procedimientos, datos y herramientas matemáticas para describir, explicar y predecir fenómenos" (p. 76). Para el Minedu-UMC (2016) "La competencia matemática es un saber actuar deliberado y reflexivo que selecciona y moviliza una diversidad de habilidades, conocimientos matemáticos, destrezas, actitudes y emociones, en la formulación y resolución de problemas en una variedad de contextos" (p. 41).

Hay una diferencia entre matemática y competencia matemática. Godino (2012, citado en Medina, 2017) dice que la matemática es un sistema de reglas (definiciones, axiomas, teoremas), que tienen una justificación fenomenológica y están lógicamente estructuradas, mientras que la competencia matemática requiere el dominio de los sistemas matemáticos disponibles y la capacidad para desarrollarlos ante las necesidades de resolver nuevos problemas (p. 38). La competencia matemática va más allá de dominar los algoritmos matemáticos, su actuar es el de saber aplicarlos de manera pertinente en la solución de situaciones planteadas. Esto implica, que el estudiante con el uso de sus conocimientos matemáticos sea capaz de afrontar una situación problemática real, para esto deberá combinar una serie de capacidades, habilidades, destrezas y actitudes a fin de darle solución. Niss (2002, citado en Arreguín, Alfaro, \& Ma, 2012) manifiesta que son ocho las competencias matemáticas: "pensar y razonar; argumentar, comunicar, modelar, plantear y resolver problemas; representar, hacer uso del lenguaje y operaciones simbólicas; utilizar ayudas y herramientas" (p.267). Para el presente estudio se ha tomado las competencias matemáticas planteadas en el currículo nacional de la educación básica 
por el Minedu: Resuelve problemas de: cantidad; de regularidad, equivalencia y cambio; de forma movimiento y localización; de gestión de datos e incertidumbre Minedu (2017).

\section{ESTRATEGIAS METODOLÓGICAS}

El presente estudio siguió el enfoque cuantitativo, porque realizó un proceso secuencial de etapas, empezando por el planteamiento del problema, revisión de literatura sustentatoria, delimitación de objetivos y preguntas que orientaron el estudio, determinó hipótesis, definió variables conceptual y operacionalmente, buscó un diseño para probar hipótesis y medir las variables, empleó métodos estadísticos para su análisis e interpretación de resultados y finalmente se obtuvo las conclusiones respondiendo a los objetivos e hipótesis planteadas (Hernández et al., 2014, p.37). También responde a las características de una investigación aplicada, porque se planteó un problema de investigación e hipótesis de trabajo, las cuales guiaron la investigación, así mismo se sustentó en un marco teórico y además concluyó en una propuesta pedagógica para responder a la problemática identificada. El método que siguió, fue el hipotético deductivo. Para Neill y Cortez (2017) es el procedimiento que inicia con el planteamiento de un problema, se formula hipótesis, se prueba las hipótesis, para luego aceptarlas o rechazarlas. Según Neill y Cortez (2017) responde a un diseño fue cuasi experimental; porque se trabajó con grupo control y grupo experimental para comparar el efecto del Aprendizaje basado en proyectos sobre las competencias matemáticas (p. 73).

\section{Población.}

Vara (2008) sostiene que "es el conjunto de individuos que tienen una o más propiedades en común, se encuentran en un espacio o territorio y varían en el transcurso del tiempo" (p.238). Para el presente estudio, la población estuvo conformada por los estudiantes del VII ciclo, quinto grado sección A y B de la institución educativa $\mathrm{N}^{\circ} 81025$ José Antonio Encinas del distrito de Víctor Larco Herrera-Trujillo; matriculados en año 2021, siendo las dos únicas secciones. Los pertenecientes a la sección A, se tomó como grupo control (27 alumnos) y los pertenecientes a la sección B, como grupo experimental (30 alumnos).

\section{Técnicas e instrumentos de recolección de datos.}

La técnica que permitió recoger los datos de la variable dependiente (competencias matemáticas) fue la encuesta. La encuesta es un conjunto de procedimientos estructurados, mediante los cuales se recolecta datos orales o escritos de una muestra 
representativa y se limita a lo que solicita el instrumento empleado (Cea, 2001, como se citó en Useche et al.,2020).

El instrumento utilizado, fue una prueba escrita de competencias matemáticas. Vara (2008) define a la prueba escrita como "situaciones controladas en la que se busca verificar el nivel de aprendizaje logrado por los estudiantes y se sustenta en criterios e indicadores de evaluación definidos previamente" (p. 290). La prueba escrita estuvo compuesta por 16 ítems dicotómicos (correcto=1, incorrecto=0). Fue aplicada de forma sincrónica, a través del aplicativo Google meet, utilizando el formulario Google. Estuvo dividida en cuatro partes: Parte 1: Competencia Resuelve problemas de cantidad, cuatro ítems (1-4). Parte 2: Competencia resuelve problemas de regularidad, equivalencia y cambio, cuatro ítems (5-8). Parte 3: Competencia resuelve problemas de forma movimiento y localización, cuatro ítems (9-12). Parte 4: Competencia resuelve problemas de gestión de datos e incertidumbre, cuatro ítems (13-16). Los niveles de logro para medir las competencias matemáticas fue nivel, inicio, nivel proceso, nivel logrado y nivel satisfactorio.

\section{Validez}

Según Hernández y Mendoza (2018) es el grado en que un instrumento mide con exactitud la variable que verdaderamente pretende medir (p. 219). Para obtener la validez de contenido, el instrumento fue sometido al criterio de un grupo de jueces expertos, constituido por cinco doctores, quienes, por unanimidad, dieron la conformidad de la estructura del instrumento. Para obtener la validez del constructo, se empleó el estadístico multivariado de análisis factorial. Para Hernández y Mendoza (2018) la validez del constructo está relacionado con la teoría, es decir, debe existir correlación entre la variable, dimensiones, ítems y la teoría planteada en el estudio. El resultado del análisis factorial que midió la competencia matemática a través de sus 16 ítems, mostró un KMO $=0.607$, el cual indica que los ítems que componen cada factor generan correspondencia entre las dimensiones planteadas. Según Kaiser, Meyer y Olkin, si el KMO $\geq 0,6$ es aceptable. Así mismo, la Prueba de esfericidad de Bartlett (Chi-cuadrado aproximado = 291,664, g.1. = 120, Sig. =0.000) indica que los ítems no se encuentran relacionados entre sí, siendo entonces significativas, por lo tanto, según este indicador, la matriz de datos resulta apropiada para realizar sobre ella la factorización. 


\section{Confiabilidad}

Según Rengel, et al. (2018, como se citó en Castro, 2020) la confiabilidad de un instrumento se da en su aplicación repetida a una misma muestra produce resultados iguales de tipo consistente y coherente. Así mismo, Hernández y Mendoza (2018) indicaron que los valores resultantes del grado de fiabilidad oscilan entre cero y uno, siendo cero, alto nivel de error y uno, 100\% confiable. La confiabilidad se calculó con el indicador de fidelidad de KUDER - RICHARDSON (KR 20) obteniendo un valor igual a 0,8079 verificándose una alta confiabilidad.

\section{Procedimiento}

Para la aplicación del instrumento, se envió solicitud a la dirección de la institución donde se realizó la investigación, luego se coordinó con los estudiantes, acordando el día y hora de la aplicación de la prueba escrita (pretest) al grupo control y experimental. La otra etapa fue la aplicación de la metodología "Aprendizaje basado en proyectos", que consistió en la realización de 22 sesiones distribuidas en tres proyectos sobre: Participación ciudadana para el fortalecimiento del sistema democrático, Toma de decisiones responsables para el emprendimiento, Desafíos como país a nuestros 200 años de vida republicana, desarrollado durante el I trimestre. Al finalizar la propuesta se aplicó la evaluación final (Post test) a ambos grupos. Finalmente se realizó el procesamiento de los resultados en una hoja de Excel y el software SPSS versión 25 para luego analizarlos en el capítulo de discusión de resultados.

\section{RESULTADOS Y DISCUCIÓN}

Tabla 1: Categorías del Pretest y Post test del Grupo Control de competencias matemáticas

\begin{tabular}{|c|c|c|c|c|c|c|c|c|c|c|c|c|c|c|c|c|c|c|c|c|c|}
\hline & & \multicolumn{10}{|c|}{ Pretest - Grupo Control } & \multicolumn{10}{|c|}{ Postes - Grupo Control } \\
\hline & & \multicolumn{2}{|c|}{ Cantidad } & \multicolumn{2}{|c|}{ Regularid } & \multicolumn{2}{|c|}{ Forma } & \multicolumn{2}{|c|}{ G. datos } & \multicolumn{2}{|c|}{$\mathrm{CM}$} & \multicolumn{2}{|c|}{ Cantidad } & \multicolumn{2}{|c|}{ Regularid } & \multicolumn{2}{|c|}{ Forma } & \multicolumn{2}{|c|}{ G. datos } & \multicolumn{2}{|c|}{$\mathrm{CM}$} \\
\hline & & $\mathrm{f}$ & $\%$ & f & $\%$ & f & $\%$ & $\mathrm{f}$ & $\%$ & f & $\%$ & $\mathrm{f}$ & $\%$ & $\mathrm{f}$ & $\%$ & $\mathrm{f}$ & $\%$ & $\mathrm{f}$ & $\%$ & f & $\%$ \\
\hline \multirow[t]{4}{*}{ Váli } & I & 9 & 30,0 & 13 & 43,3 & 13 & 43,3 & 22 & 73,3 & 6 & 20,0 & 8 & 26,7 & 12 & 40,0 & 11 & 36,7 & 15 & 50,0 & 2 & 6,7 \\
\hline & $\mathrm{P}$ & 12 & 40,0 & 6 & 20,0 & 12 & 40,0 & 4 & 13,3 & 16 & 53,3 & 12 & 40,0 & 7 & 23,3 & 12 & 40,0 & 9 & 30,0 & 20 & 66,7 \\
\hline & $\mathrm{L}$ & 6 & 20,0 & 3 & 10,0 & 2 & 6,7 & 1 & 3,3 & 5 & 16,7 & 7 & 23,3 & 4 & 13,3 & 4 & 13,3 & 2 & 6,7 & 5 & 16,7 \\
\hline & $S$ & 0 & 0 & 5 & 16,7 & 0 & 0 & 0 & 0 & 0 & 0 & 0 & 0 & 4 & 13,3 & 0 & 0 & 1 & 3,3 & 0 & 0 \\
\hline \multirow[t]{3}{*}{ Per } & $\mathrm{T}$ & 27 & 90,0 & 27 & 90,0 & 27 & 90,0 & 27 & 90,0 & 27 & 90,0 & 27 & 90,0 & 27 & 90,0 & 27 & 90,0 & 27 & 90,0 & 27 & 90,0 \\
\hline & $\mathrm{S}$ & 3 & 10,0 & 3 & 10,0 & 3 & 10,0 & 3 & 10,0 & 3 & 10,0 & 3 & 10,0 & 3 & 10,0 & 3 & 10,0 & 3 & 10,0 & 3 & 10,0 \\
\hline & Tota & 30 & 100,0 & 30 & 100,0 & 30 & 100,0 & 30 & 100,0 & 30 & 100,0 & 30 & 100,0 & 30 & 100,0 & 30 & 100,0 & 30 & 100,0 & 30 & 100,0 \\
\hline
\end{tabular}

Nota. Data de Competencia matemática. I=inicio; $\mathrm{P}=$ proceso; $\mathrm{L}=\log$ rado; $\mathrm{S}=$ satisfactorio Interpretación

En el pre test del grupo control se visualiza que la mayor parte de estudiantes se encuentran ubicado en el nivel proceso $53,3 \%$ y solo el $16,7 \%$ se encuentra en el nivel 
logrado; mientras que en el post test del mismo grupo se observa que hubo un aumento del 13,4\% en el nivel proceso y no hubo variación en nivel logrado y satisfactorio.

Tabla 2: Categorías del Pretest y Post test del Grupo Experimental de competencias matemáticas

\begin{tabular}{|c|c|c|c|c|c|c|c|c|c|c|c|c|c|c|c|c|c|c|c|c|c|}
\hline \multicolumn{12}{|c|}{ Pretest - Grupo experimental } & \multicolumn{10}{|c|}{ Postest - Grupo Experimental } \\
\hline & & \multicolumn{2}{|c|}{ Cantid } & \multicolumn{2}{|c|}{ Regular } & \multicolumn{2}{|c|}{ Forma } & \multicolumn{2}{|c|}{ G. datos } & \multicolumn{2}{|c|}{$\mathrm{CM}$} & \multicolumn{2}{|c|}{ Cantid } & \multicolumn{2}{|c|}{ Regular } & \multicolumn{2}{|c|}{ Forma } & \multicolumn{2}{|c|}{ G.datos } & \multicolumn{2}{|c|}{$\mathrm{CM}$} \\
\hline & & $\mathrm{f}$ & $\%$ & $\mathrm{f}$ & $\%$ & $\mathrm{f}$ & $\%$ & $\mathrm{f}$ & $\%$ & f & $\%$ & $\mathrm{f}$ & $\%$ & $\mathrm{f}$ & $\%$ & $\mathrm{f}$ & $\%$ & $\mathrm{f}$ & $\%$ & $\mathrm{f}$ & $\%$ \\
\hline \multirow[t]{4}{*}{ Váli } & I & 11 & 36,7 & 12 & 40,0 & 17 & 56,7 & 19 & 63,3 & 6 & 20,0 & 2 & 6,7 & 0 & 6,7 & 3 & 10,0 & 3 & 10,0 & 0 & 0 \\
\hline & $P$ & 11 & 36,7 & 8 & 26,7 & 9 & 30,0 & 7 & 23,3 & 20 & 66,7 & 5 & 16,7 & 8 & 26,7 & 7 & 23,3 & 11 & 36,7 & 5 & 16,7 \\
\hline & $\mathrm{L}$ & 7 & 23,3 & 7 & 23,3 & 3 & 10,0 & 3 & 10,0 & 4 & 13,3 & 19 & 63,3 & 16 & 53,3 & 16 & 53,3 & 11 & 36,7 & 18 & 60,0 \\
\hline & $S$ & 1 & 3,3 & 3 & 10,0 & 1 & 3,3 & 1 & 3,3 & 0 & 0 & 4 & 13,3 & 4 & 13,3 & 4 & 13,3 & 5 & 16,7 & 7 & 23,3 \\
\hline $\begin{array}{c}\text { Per } \\
\text { d }\end{array}$ & Sist. & 0 & 0 & 0 & 0 & 0 & 0 & 0 & 0 & 0 & 0 & 0 & 0 & 0 & 0 & 0 & 0 & 0 & 0 & 0 & 0 \\
\hline & $\begin{array}{c}\text { Tot } \\
\mathrm{a}\end{array}$ & 30 & $\begin{array}{c}100 \\
0\end{array}$ & 30 & $\begin{array}{c}100 \\
0\end{array}$ & 30 & $\begin{array}{c}100, \\
0\end{array}$ & 30 & $\begin{array}{c}100, \\
0\end{array}$ & 30 & $\begin{array}{c}100, \\
0\end{array}$ & 30 & $\begin{array}{c}100, \\
0\end{array}$ & 30 & $\begin{array}{c}100 \\
0\end{array}$ & 30 & $\begin{array}{c}100 \\
0\end{array}$ & 30 & $\begin{array}{c}100 \\
0\end{array}$ & 30 & $\begin{array}{c}100, \\
0\end{array}$ \\
\hline
\end{tabular}

Nota. Data de Competencia matemática. $\mathrm{I}=$ inicio; $\mathrm{P}=$ proceso; $\mathrm{L}=\operatorname{logrado} ; \mathrm{S}=$ satisfactorio

\section{Interpretación}

En el pretest del grupo experimental se observa que la mayor parte de estudiantes se encuentran ubicado en el nivel proceso $(66,7 \%)$ y en el post test del mismo grupo se observa que ha disminuido el porcentaje en el nivel proceso para aumentar en el nivel logrado en 46\%, del mismo modo en el nivel satisfactorio aumentó en un 23,3\%.

Tabla 3: Estadígrafos Pretest - Post test - Grupo Control y experimentales competencias matematicas

\begin{tabular}{|c|c|c|c|c|c|c|c|c|c|c|c|c|c|c|c|c|c|c|c|c|}
\hline & \multicolumn{5}{|c|}{$\begin{array}{c}\text { Pre-test } \\
\text { Grupo experimental }\end{array}$} & \multicolumn{5}{|c|}{$\begin{array}{c}\text { Pre-test } \\
\text { Grupo control }\end{array}$} & \multicolumn{5}{|c|}{$\begin{array}{c}\text { Post test } \\
\text { Grupo experimental }\end{array}$} & \multicolumn{5}{|c|}{$\begin{array}{c}\text { Post test } \\
\text { Grupo control } \\
\end{array}$} \\
\hline & DC & DR & DF & DG & $\mathrm{CM}$ & DC & DR & DF & DG & $\mathrm{CM}$ & DC & DR & DF & DG & $\mathrm{CM}$ & DC & DR & DF & DG & $\mathrm{CM}$ \\
\hline Válid & 30 & 30 & 30 & 30 & 30 & 27 & 27 & 27 & 27 & 27 & 30 & 30 & 30 & 30 & 30 & 27 & 27 & 27 & 27 & 27 \\
\hline N Perdi & 0 & 0 & 0 & 0 & 0 & 3 & 3 & 3 & 3 & 3 & 0 & 0 & 0 & 0 & 0 & 3 & 3 & 3 & 3 & 3 \\
\hline Media & 1,90 & 1,97 & 1,33 & 1,33 & 6,53 & 1,78 & 1,85 & 1,48 & 1,04 & 6,15 & 2,83 & 2,70 & 2,70 & 2,57 & 10,80 & 1,85 & 1,85 & 1,63 & 1,41 & 6,74 \\
\hline Mediana & 2,00 & 2,00 & 1,00 & 1,00 & 6,00 & 2,00 & 2,00 & 2,00 & 1,00 & 6,00 & 3,00 & 3,00 & 3,00 & 3,00 & 11,00 & 2,00 & 2,00 & 2,00 & 1,00 & 7,00 \\
\hline Moda & 2 & 1 & $1 \mathrm{a}$ & 1 & $5 \mathrm{a}$ & 2 & 1 & 2 & 1 & $5 \mathrm{a}$ & 3 & 3 & 3 & $2 \mathrm{a}$ & 11 & 2 & 1 & 2 & 1 & $5 \mathrm{a}$ \\
\hline Desv. & ,923 & 1,129 & 1,093 & 1,028 & 2,389 & ,934 & 1,350 & 802 & ,706 & 2,332 & ,747 & 877, & ,837 & ,971 & 2,107 & ,949 & 1,292 & ,884 & 1,010 & 1,852 \\
\hline Mínimo & 0 & 0 & 0 & 0 & 3 & 0 & 0 & 0 & 0 & 1 & 1 & 0 & 1 & 0 & 5 & 0 & 0 & 0 & 0 & 3 \\
\hline Máximo & 4 & 4 & 4 & 4 & 12 & 3 & 4 & 3 & 3 & 10 & 4 & 4 & 4 & 4 & 14 & 3 & 4 & 3 & 4 & 10 \\
\hline
\end{tabular}

a. Existen múltiples modos. Se muestra el valor más pequeño.

Nota. Data de Competencia matemática. DC=dimensión cantidad; DR=dimensión regularidad; $\mathrm{DF}=$ dimensión forma; $\mathrm{DG}=$ dimensión gestión de datos.

\section{Interpretación}

Los valores de la media en el grupo control del pretest y post test de la competencia matemática son parecidos 6,15 y 6,74 respectivamente, ubicándose en el nivel de logro 
proceso; mientras que entre el grupo experimental del pretest y del post test de la misma variable, se muestra una diferencia entre sus medias de 4,27 unidades; logrando pasar de un nivel logro en proceso a destacado después de haber recibido las sesiones con el método ABP.

\section{Pruebas de normalidad}

Se realizó para comprobar si los resultados siguen una distribución normal, para ello se consideró el tamaño de la muestra para elegir la prueba correspondiente. Por ser muestra $\mathrm{n}=30<50$ se eligió el test de Shapiro- Wilk. Los datos son contrastados con el nivel de significancia para la toma de decisión en la elección del test apropiado para realizar la prueba de hipótesis.

Los resultados fueron que la mayoría de valores Sig. de la Competencia Matemática y sus dimensiones en pre y Postest de los grupos Control y Experimental, fueron < 0.05, lo que evidenció una distribución no paramétrica, por ello para la Prueba de Hipótesis se usó el Test U de Mann Whitney.

\section{Prueba de hipótesis}

Ho: El aprendizaje basado en proyectos no impacta positiva y significativamente en las competencias matemáticas de los estudiantes del quinto grado del nivel secundario de la Institución Educativa José Antonio Encinas de Trujillo, 2021.

Hi: El aprendizaje basado en proyectos impacta positiva y significativamente en las competencias matemáticas de los estudiantes del quinto grado del nivel secundario de la Institución Educativa José Antonio Encinas de Trujillo, 2021.

Tabla 4: Prueba de hipótesis Postest competencias matemáticas Grupo control y experimental

\begin{tabular}{|c|c|c|c|c|c|}
\hline & $\begin{array}{l}\text { D. Cant } \\
\text { GC_GE }\end{array}$ & $\begin{array}{c}\text { D. Regul. } \\
\text { GC_GE }\end{array}$ & $\begin{array}{l}\text { D. Forma } \\
\text { GC_GE }\end{array}$ & $\begin{array}{c}\text { D. Gest. Dat. } \\
\text { GC_GE }\end{array}$ & $\begin{array}{c}\text { VCM } \\
\text { GC_GE }\end{array}$ \\
\hline $\begin{array}{c}\text { Ude Mann- } \\
\text { Whitney }\end{array}$ & 174,500 & 240,000 & 162,000 & 165,500 & 64,500 \\
\hline $\begin{array}{c}\text { W de } \\
\text { Wilcoxon }\end{array}$ & 552,500 & 618,000 & 540,000 & 543,500 & 442,500 \\
\hline Z & $-3,935$ & $-2,732$ & $-4,066$ & $-3,962$ & $-5,478$ \\
\hline $\begin{array}{c}\text { Sig. } \\
\text { as intótica(bila } \\
\text { teral) } \\
\end{array}$ & ,000 & ,006 & ,000 &, 000 & ,000 \\
\hline
\end{tabular}

a. Variable de agrupación: Grupo

Note. Data de Competencia matemática. VCM= variable competencia matemática. 


\section{Interpretación}

La variable competencia matemáticas del grupo control y experimental en el post test muestran un U- Mann-Whitney=64,500 y $Z=-5,478 ; p=0,00<0,05$ por lo cual se rechaza la Ho y se acepta la Hi, concluyendo que la variable competencias matemáticas del grupo experimental muestra mejoras significativas con respecto al grupo control, entonces, la influencia es altamente significativa del Aprendizaje basado en Proyectos en las Competencias matemáticas. Lo mismo se puede afirmas con sus dimensiones; así lo demuestra los valores Sig. P: $<0.01$.

Tabla 5: Prueba Hipótesis Pre test - Postest - Grupo experimental Competencias matemáticas

\begin{tabular}{ccccccc}
\hline & $\begin{array}{c}\text { Postest-Pretest } \\
\text { D. Can. }\end{array}$ & $\begin{array}{c}\text { Postest-pretest } \\
\text { D. Reg. }\end{array}$ & $\begin{array}{c}\text { Postest-pretest } \\
\text { D. Forma }\end{array}$ & $\begin{array}{c}\text { Postest-pretest } \\
\text { D. Ges. }\end{array}$ & $\begin{array}{c}\text { VCM-Post } \\
\text { VCM-Pret }\end{array}$ \\
\hline $\mathrm{Z}$ & $\begin{array}{c}- \\
\text { V }\end{array}$ & $-3,508^{\mathrm{b}}$ & $-3,968^{\mathrm{b}}$ & $-4,344^{\mathrm{b}}$ & $-4,715^{\mathrm{b}}$ \\
\hline $\begin{array}{c}\text { Sig. } \\
\text { asintótica } \\
\text { (bilateral) }\end{array}$ &, 000 &, 000 &, 000 &, 000 &, 000 \\
\hline
\end{tabular}

a. Prueba de rangos con signo de Wilcoxon

b. Se basa en rangos negativos.

Nota. Data de Competencia matemática

\section{Interpretación:}

En la prueba de rangos con signo de Wilcoxon, la variable competencia matemáticas del grupo experimental en el pre test y post test muestran $Z=-4,715 ; p=0,00<0,05$ por lo cual se rechaza la Ho y se acepta la Hi, concluyendo que la variable competencias matemáticas del grupo experimental en el pre test muestra mejoras significativas con respecto al Postest, por lo tanto, la influencia es altamente significativa del Aprendizaje basado en Proyectos en las Competencias matemáticas. Lo mismo se afirma con sus dimensiones; así lo demuestra los valores Sig. P: $<0.01$.

\section{DISCUSIÓN}

La finalidad de este estudio fue determinar el efecto del aprendizaje basado en proyectos mediados por TIC en el desarrollo de competencias matemáticas en los estudiantes del quinto grado del nivel secundario de la institución educativa 81025 José Antonio Encinas de Trujillo, En esta sección se analizaron e interpretaron los resultados obtenidos en el marco de los antecedentes y las bases teóricas de las variables desde cada uno de sus componentes. 
Con respecto a los resultados del pre test del grupo control y grupo experimental no hubo variación significativa. En el grupo control se encontró: nivel inicio un $20 \%$ de estudiantes, en nivel proceso $53 \%$, en nivel logrado $16,7 \%$ y en el nivel satisfactorio $0 \%$. De igual forma se obtuvo los resultados en el grupo experimental; siendo, en nivel inicio $20 \%$ de estudiantes, nivel proceso $66,7 \%$, en nivel logrado $13,3 \%$ y nivel satisfactorio $0 \%$. Como se aprecia en el nivel inicio y satisfactorio no hubo ninguna variación (inicio $20 \%$ y satisfactorio 0\%), mientras en nivel proceso y logrado hubo ligera diferencia 13,7\% y 3,4\% respectivamente. Estos hallazgos coinciden con Flores y Juárez (2017), quienes, en su trabajo titulado: "Aprendizaje basado en proyectos para el desarrollo de competencias matemáticas en Bachillerato", aplicaron su estudio en estudiantes de las mismas edades (15- 17 años), en una muestra pequeña (32 estudiantes), utilizaron niveles de logro parecidos para evaluar la competencia (Pre formal, receptivo, resolutivo y estratégico); antes de aplicar el método ABP se obtuvo resultados de: 63\% de los estudiantes se encontraron en el nivel pre formal y el 36\% restante apenas en el receptivo. Los aprendices se limitaron a realizar las tareas solicitadas, no revelaron autonomía ni iniciativa, se mostraron limitados para realizar un análisis más profundo. Ambos resultados nos muestran que no importa el lugar geográfico donde se ubica la escuela, la realidad es que los estudiantes no lograron desarrollar competencias matemáticas con las estrategias tradicionales de enseñanza que emplean los docentes.

Con respecto al Postest después de realizar las 22 sesiones con la aplicación del “Aprendizaje basado en proyectos", solo al grupo experimental y el grupo control también recibió sesiones, pero con el método tradicional los resultados fueron: en el grupo control, en inicio $6,7 \%$ de estudiantes, en el nivel proceso 66\%, nivel logrado $16 \%$ y nivel satisfactorio 0\%; no encontrándose variación significativa. En cambio, en el grupo experimental, si hubo resultados positivos y altamente significantes. En el nivel inicio $0 \%$, nivel proceso $16 \%$ y en el nivel logrado $60 \%$ y nivel satisfactorio $23,3 \%$; concluyendo que el aprendizaje basado en proyectos mediados por TIC, influye significativamente en el desarrollo de las competencias matemáticas de los estudiantes del quinto grado del nivel secundario de la institución educativa José Antonio Encinas de Trujillo. Estos hallazgos también se compararon con Flores y Juárez (2017), quienes después de haber aplicado la estrategia Aprendizaje basado en proyectos para el desarrollo de competencias matemáticas, encontraron un resultado altamente 
significativo ya que el $90 \%$ de la muestra se encontraron en el nivel estratégico y solo el $10 \%$ restante en el nivel resolutivo, indicando un gran avance en el desempeño de los estudiantes. Esto hace inferir que el método de aprendizaje basado en proyectos influye significativamente en el desarrollo de competencias matemáticas.

Los resultados obtenidos en el Postest de los estudiante de la Institución educativa José Antonio Encinas, es importante resaltar, porque a pesar de los escasos recursos tecnológicos usados durante el desarrollo del proyecto, por la situación económica de las familias, por la situación social afectivo a consecuencia de la pandemia ocasionada por la Covid 19, mostraron desarrollar sus competencias matemáticas utilizando TIC, además, se volvieron creativos al presentar sus evidencias de aprendizaje y más confiados en expresarlas usando redes sociales como el blog, además, incrementó su motivación por las Matemáticas. Esto implica la existencia positiva y significativa del aprendizaje basado en proyectos con respecto a las competencias matemáticas.

Ante esta situación, se afirma que el aprendizaje basado en proyectos es un método que cumple con las características para desarrollar competencias y que actualmente es el enfoque de todo acto educativo. Así como lo menciona Maldonado (2018) que este método fomenta la integración sistémica de competencias: saber conocer, saber hacer, saber ser y saber convivir (p. 172). También el Instituto Tecnológico y de estudios superiores de Monterrey (s.f) sostiene que el aprendizaje a través de proyectos permite a los estudiantes ser responsables de su propio aprendizaje, desencadenando en ellos el amor por aprender, considerándose actores de cambio al aplicar lo que aprendieron en la solución de situaciones problematizadoras (p.3).

Con respecto a la dimensión resuelve problemas de cantidad en el pretest y Postest del grupo experimental, los resultados evidenciaron que hubo un progreso significativo, porque, en el nivel inicio del 36\% detectado en el pretest disminuyó al 6\% en el Postest. En el nivel proceso de $36 \%$ bajó a $16 \%$ en el Postest. En nivel logrado de 23,3 \% en el pretest aumentó a 63,3\% en el Postest y en el nivel satisfactorio de un 3,3\% en el pretest aumentó a 13,3\% en el Postest. Estos resultados demuestran la influencia directa y altamente significativa de la estrategia $\mathrm{ABP}$ en esta dimensión, corroborando que el aprendizaje basado en proyectos mediados por TIC, influye significativamente en esta dimensión. Así como Vargas et al. (2020), en su estudio “Aprendizaje basado en proyectos mediados por TIC para superar dificultades en el aprendizaje de las operaciones 
básicas matemáticas", emplearon la estrategia Aprendizaje basado en proyectos en dos grupos de estudiantes de manera simultánea, grupo A y grupo B. La diferencia con el presente estudio fue que a ambos grupos se aplicó la estrategia Aprendizaje basado en proyectos, solo que en el grupo A se desarrolló el proyecto empleando material educativo computarizado, mientras que en el grupo B lo hizo con folletos escritos. Los resultados fueron: En el pretest con desempeño bajo $27 \%$, básico $66 \%$ y superior 7\%. En el test final se observa el notable avance en la superación de dificultades matemáticas, ya que los estudiantes obtuvieron en nivel básico un 17\%, y superior el 83\%.Los resultados de la investigación arrojaron que solo la diferencia entre ambos grupos, es el tiempo, que los proyectos desarrollados utilizando las TIC ayudan a mejorar la presentación y optimizar el tiempo; pero el resultado en ambos grupos arrojó la mejora en los aprendizajes de la matemática, además se visualizó en los estudiantes su autorregulación y autocontrol con respecto a sus actitudes positivas ante la matemática

Con respecto a la dimensión resuelve problemas de regularidad equivalencia y cambio en el pretest y Postest del grupo experimental, los resultados evidenciaron que del $100 \%$ de la muestra, en el nivel inicio del $40 \%$ obtenido en el pretest disminuyó a 6,7\% en el Postest. En el nivel proceso no hubo variación (26,7\%). En el nivel logrado en el pretest de 23,3\% aumento a 53,3\% en el Postest y en el nivel satisfactorio de $10 \%$ en el pretest pasó a 13,3\% en el Postest, probándose que el aprendizaje basado en proyectos mediados por TIC, influye significativamente en este componente. Los hallazgos de Alvis et al. (2019), en su investigación titulada: Los ambientes de aprendizaje reales como estrategia pedagógica para el desarrollo de competencias matemáticas, establece una relación positiva con estos resultados, porque a pesar de ser una investigación de tipo cualitativo, con enfoque comprensivo, su objetivo fue describir, interpretar y comprender ambientes reales que sirvan como estrategias para fomentar una apreciación crítica, argumentativa; propia de la competencia matemática. Los resultados verificaron que los estudiantes después de visitar y estudiar los ambientes seleccionados pudieron crear sus propias situaciones problemáticas, comprenderlos y resolverlos con mínima dificultad, empleando estrategias heurísticas. La similitud de ambos trabajos es que tuvieron como fin desarrollar competencias, ambos buscaron ambientes reales, para operabilizar y actuando en dichos ambientes incrementaron su motivación por aprender, la autonomía, la autorregulación y desarrollo de competencias matemáticas. 
También, con respecto a la dimensión resuelve problemas de forma movimiento y localización en el pretest y Postest del grupo experimental, los resultados evidenciaron que del $100 \%$ de la muestra, se obtuvo en el nivel inicio del pretest de un $56,7 \%$ disminuyó considerablemente a $10 \%$ en el Postest. En el nivel proceso del pretest $40 \%$ disminuyó a 23,3\% en el Postest. En el nivel logrado de $10 \%$ en el pretest aumento significativamente a 53,3\% en el Postest y en el nivel satisfactorio de 3,3\% en el pretest pasó a 13,3\% en el Postest. De estos resultados se concluye que el aprendizaje basado en proyectos mediados por TIC, influye significativamente en esta dimensión. Estos resultados, corroboran lo manifestado por Barrera (2017), quien sostiene que el aprendizaje mediante proyectos usado en el proceso de la enseñanza y el aprendizaje permite al estudiante acrecentar su motivación por aprender, a conectarse entre la escuela y su realidad, ir de la teoría a la práctica, aprender a trabajar colaborativamente. Así mismo, le permite desarrollar habilidades blandas, le ofrece la oportunidad de proyectarse a su comunidad, proponiendo y desarrollando proyectos simples y complejos, de corta, mediano y largo plazo, le ayuda a mejora su autoestima, le permite auto regular su aprendizaje, siendo consciente de lo que necesita aprender, conocer y desaprender para volver aprender.

Así mismo, con respecto a la dimensión resuelve problemas de gestión de datos e incertidumbre en el pretest y Postest del grupo experimental, los resultados fueron positivos y altamente significativos. En el nivel inicio del 63,3\% en el pretest bajó considerablemente a $10 \%$ en el Postest. En el nivel proceso del 23,3\% en el pretest aumentó a 36,7\% en el Postest. En el nivel logrado de 10\% en el pretest aumento a 36,7\% en el Postest y en el nivel satisfactorio de 3,3\% en el pretest pasó a 16,7\% en el Postest; probándose que el aprendizaje basado en proyectos mediados por TIC, influye significativamente en este componente. Esta conclusión es corroborada totalmente por Barrera (2017) en su estudio: Impacto del Aprendizaje basado en proyectos colaborativos ABPC, mediados por la Tecnología de la información y comunicación TIC, para el desarrollo de competencias en estadística en el cual concluyó que la estrategia del aprendizaje basado en el ABPC, contribuye significativamente en la mejora de las competencias en estadística. Además, la inclusión de las TIC permite a los estudiantes aprender nuevas herramientas informáticas, favoreciendo así su formación académica. Estos resultados también son confirmados por Inzunza (2017), quien, en su investigación 
de enfoque cualitativo, sobre el potencial que tienen los proyectos para desarrollar la motivación y las competencias de razonamiento y pensamiento estadístico, realizado en 19 estudiantes de 19 a 21 años, y utilizando para el recojo de información una prueba de actitudes pudo demostrar, que los proyectos tienen un alto potencial para desencadenar actitudes positivas hacia la estadística y darle un sentido de utilidad para resolver situaciones reales.

Del mismo modo, los resultados indican que los grupos que no recibieron la estrategia del aprendizaje basado en proyectos mantuvieron un promedio similar: grupo control pretest 6,15; grupo control Postest 6,74; así como el grupo experimental pretest 6,53; mientras que el grupo experimental post test 10,80. También se visualiza que en el pretest en ambos grupos el 50\% contestó menos de 6 ítems correctos y el post test el grupo control contestó menos de 7 ítems correctos. El grupo experimental post test alcanzó una mediana de 11. Estos resultados demuestran que los estudiantes que continuaron desarrollando sus aprendizajes con estrategias tradicionales no mejoraron en el desarrollo de competencias matemáticas; mientras que el grupo que recibió la estrategia aprendizaje basado en proyectos durante el tiempo previsto y con las fases de la misma estrategia tuvieron un avance en su promedio de 6,53 a 10,80 y una mediana de 6,00 a 11,00; ubicándose en el nivel esperado. Estos resultados vuelven a corroborar que la estrategia ABP, influye

significativamente en el desarrollo de competencias matemáticas. Estos resultados se asemejan al estudio de Vargas et al. (2020), quienes en su estudio aprendizaje basado en Proyectos mediados por tic para superar dificultades en el aprendizaje de operaciones básicas matemáticas, con diseño cuasi experimental, concluyeron que en el grupo experimental pretest el 50\% de estudiantes obtuvieron 20 errores o menos y en el Postest del mismo grupo el $50 \%$ comete 5 errores o menos de 30 ítems que tuvo la prueba. De este modo, se corrobora que el aprendizaje basado en proyectos mejora eficazmente el desarrollo de competencias matemáticas.

\section{CONSIDERACIONES FINALES}

La presente investigación se propuso como objetivo proponer una metodología basada en proyectos para mejorar las competencias matemáticas en los estudiantes del quinto grado del nivel secundario de la institución educativa José Antonio Encinas de Trujillo. Este objetivo se logró en su totalidad, ya que los estudiantes después de la aplicación de las sesiones programadas evidenciaron mejoras en las competencias matemáticas tal como 
se observa en las tablas analizadas. Así mismo se logró concluir con los objetivos específicos trazados: Determinar de qué manera el aprendizaje basado en proyectos mediados por TIC, influye en la competencia cantidad, regularidad equivalencia y cambio, forma, movimiento y localización, gestión de datos e incertidumbre de los estudiantes del quinto grado del nivel secundario de la institución educativa José Antonio Encinas de Trujillo, concluyendo que su influencia es altamente significativa.

\section{REFERENCIAS}

Alvis-Puentes, J., Aldana-Bermúdez, E. y Caicedo-Zambrano, S. (2019, agosto). Los ambientes de aprendizaje reales como estrategia pedagógica para el desarrollo de competencias matemáticas en estudiantes de básica secundaria. Revista de investigación, Desarrollo e Innovación, 10(1), 135-147. Disponible en: http://dx.doi.org/10.19053/20278306.v10.n1.2019.10018.

Alvites-Huamaní, C. (2017, junio). Herramientas Tic en el aprendizaje en el área de Matemática. Caso: Escuela Pop Up. Hamut'ay, 4(1),18-30. Disponible en: http://revistas.uap.edu.pe/ojs/index.php/HAMUT/article/view/1393/1358

Arias, W., Oblitas, A. (2014). Aprendizaje por descubrimiento vs. Aprendizaje significativo: Un experimento en el curso de historia de la psicología. Boletín Academia Paulista de Psicología, 34(87), 455-471. Disponible en: https://www.redalyc.org/pdf/946/94632922010.pdf

Arreguin, L., Alfaro, J., Ramírez, M. (2012). Desarrollo de competencias matemáticas en secundaria usando la técnica de aprendizaje orientado en proyectos. Reice, 10(4), 264-284. Disponible en: https://www.redalyc.org/pdf/551/55124841017.pdf

Ayerbe López, J. y Perales Palacios F. (2020, junio). "Reinventa tu ciudad": Aprendizaje basado en proyectos para la mejora de la conciencia ambiental en estudiantes de secundaria. Enseñanza de las ciencias, 38(2), 181-203. Disponible en: https://ddd.uab.cat/pub/edlc/edlc_a2020v38n2/edlc_a2020v38n2p181.pdf

Barrera Meza, M., Fernández Morales, F. y Enrique Duarte, J. (2017). Aprendizaje Basado en Proyectos colaborativos mediados por Tic para el desarrollo de competencias en Estadística. Saber, Ciencia y Libertad, 12(2), p. 220-232. Disponible en: https://revistas.unilibre.edu.co/index.php/saber/article/view/1590/1175 
Batista Monsalve, O. (2018). Aprendizaje basado en Proyectos como estrategia pedagógica para mejorar el razonamiento geométrico en los estudiantes del sexto C de la institución educativa colegio municipal María Concepción Loperena de Cúcuta [Trabajo de grado presentado como requisito para optar el título de Magister en educación, Universidad Autónoma de Bucaramanga UNAB]. Disponible en: https://repository.unab.edu.co/bitstream/handle/20.500.12749/2486/2018_Tesis Bautista_Monsalve_Olga_Cecilia.pdf?sequence $=1 \&$ isAllowed $=y$

Boillos Pereira, M. (2019, octubre). Alfabetización académica temprana a través del aprendizaje basado en Proyectos. Universidad Politécnica de Valencia, 50, 143254. DOI: https://doi.org/10.4995/lyt.2019.11421

Castro Vargas, C. (2020). Impacto del aprendizaje basado en proyectos en las competencias de redes y comunicaciones I en estudiantes de ingeniería, Lima 2020 [Tesis para obtener el grado de doctor, Universidad César Vallejo). Disponible en: https://repositorio.ucv.edu.pe/handle/20.500.12692/49473

Calduch Cervera, R. (2014). Métodos y técnicas de investigación internacional. Universidad Complutense de Madrid. Disponible en: https://www.ucm.es/data/cont/docs/835-2018-03-01-

Metodos\%20y\%20Tecnicas\%20de\%20Investigacion\%20Internacional\%20v2.pd $\underline{f}$

Cázarez Meza, M. (2015). Competencias matemáticas de los estudiantes del instituto de Valladolid. preparatoria de Morelia como aspirantes universitarios. [Tesis doctoral, Universidad de Oviedo]. Disponible en: file:///C:/Users/Mini\%20OS/Downloads/TD_MarioAlbertoCazarezMeza\%20(1). pdf

Cháves Salas, A. (2001). Implicancias educativas de la teoría sociocultural de Vigotsky. Educación. Universidad de Costa Rica, 5(22), 59-65. Disponible en: https://www.uv.mx/personal/yvelasco/files/2012/08/Implicaciones_edcucativas de_la_teoria_sociocultural_de_Vigotsky.pdf

Cisneros Gallo, T. (1996). Fundamentos de los métodos activos en la enseñanza de la línea de Filosofía y CC. SS en la educación secundaria. Educación, 5(10), p. 149- 
170.

Recuperado

a

partir

http://revistas.pucp.edu.pe/index.php/educacion/article/view/5111.

Corbalán, F. (1995, diciembre). La Matemática aplicada a la vida cotidiana. Educación Matemática, 10(3),148-150. Disponible en: http://www.revista-educacionmatematica.org.mx/descargas/Vol10/3/13Corbalan.pdf

De la Puente, M., Guerra, D., de Oro, C. y McGarry, C. (2019). Undergraduate students' perceptions of Project-Based Learning (PBL) effectiveness: A case report in the Colombian Caribbean. Cogent Education, 6(1), 1616364, pp1 - 17. Recuperado de https://www.cogentoa.com/article/10.1080/2331186X.2019.1616364.

DOI: https://doi.org/10.1080/2331186X.2019.1616364

Eichholtz, J., Jogiparthi, V., Seeling, P., Hinton, Q. y Johnson, M. (2019). Supporting Project-Based Learning through Economical and Flexible Learning Spaces. Educ. Sci. 2019, 9(3), 212. Recuperado de https://www.mdpi.com/2227-7102/9/3/212. DOI: https://doi.org/10.3390/educsci9030212

Espejo Leupin, R. (2016). ¿Pedagogía activa o métodos activos? El caso del aprendizaje activo en la Universidad. Revista digital de investigación en docencia universitaria, 10(1), 16-27. Doi http://dx.doi.org/10.19083/ridu.10.456

Esteban Nieto, N. (2018). Tipos de investigación. Universidad Santo Domingo de Guzmán. Concytec. 1-4. Disponible en:

http://repositorio.usdg.edu.pe/bitstream/USDG/34/1/Tipos-de-Investigacion.pdf

Flores-Fuentes, G. y Juárez-Ruiz, E. (2017). Aprendizaje basado en proyectos para el desarrollo de competencias matemáticas en Bachillerato. Revista Electrónica de Investigación Educativa, 19(3), 71-91. Disponible en: https://doi.org/10.24320/redie.2017.19.3.721

Garay Leiva, T. (2019). Aprendizaje basado en proyectos y la competencia indaga en estudiantes de estudiantes del 4to año de educación secundaria del colegio $N^{\circ}$ 1220 SJM [Trabajo de investigación para optar el grado académico de bachiller en educación, Universidad San Ignacio de Loyola]. Disponible en: http://repositorio.usil.edu.pe/bitstream/USIL/9713/1/2019_Garay-Leyva.pdf

García Santamaría, C. (2018, mayo). Metodología ABP en las clases de Matemática de la ESO [Trabajo final de master de profesor en educación secundaria obligatoria y bachillerato, Universidad de Valladolid]. 
http://uvadoc.uva.es/bitstream/handle/10324/31171/TFMG821.pdf?sequence=1 \&isAllowed $=\mathrm{y}$

Gavidia Medrano, J. (2018, julio). Método de Resolución de problemas y desarrollo de competencias en el área de Matemática en estudiantes de educación secundaria. Redalyc.org: Horizonte de la Ciencia, 8(15). Disponible en: https://www.redalyc.org/jatsRepo/5709/570960688008/html/index.html

Hernández Sampieri, R., Fernández Collado, C. y Batista Lucio, P. (2014). Metodología de la investigación. Sexta edición. Editorial McGraw Hill. Disponible en: https://www.uca.ac.cr/wp-content/uploads/2017/10/Investigacion.pdf

Hernández Sampieri, R., Mendoza Torres, C. (2018). Metodología de la investigación. Las rutas cuantitativas, cualitativas y mixtas. Editorial McGraw Hill.

Huanca Velarde, L. y Moreno Llacza, A. (2019, marzo). Factores asociados al rendimiento en matemática de los estudiantes del quinto grado de secundaria del departamento de Lima. Brazilian Journal of Development, 5(4), 3839-3855. Disponible

en: https://www.brazilianjournals.com/index.php/BRJD/article/view/1556/1434

Huysken, K., Olivey, H., McElmurry, K., Gao, M. y Avis, P. (2019). Assessing Collaborative, Project-based Learning Models in Introductory Science Courses. Journal of the Scholarship of Teaching and Learning, 19(1), 6-28. Recuperado de https://scholarworks.iu.edu/journals/index.php/josotl/article/view/26777

https://doi.org/10.14434/josotl.v19i1.26777

Instituto tecnológico y de estudios superiores de Monterrey. (2010). Investigación e innovación educativa. Centro virtual de técnicas didácticas. Disponible en: http://sitios.itesm.mx/va/dide2/tecnicas_didacticas/aop/copaop.htm

Instituto tecnológico y de estudios superiores de Monterrey. (s.f). Las estrategias y técnicas didácticas en el rediseño. El método de proyectos como técnica didáctica. Vicerrectoría académica. Instituto tecnológico y de estudios superiores de Monterrey. Disponible en: http://sitios.itesm.mx/va/dide2/documentos/proyectos.PDF

Liu, M. (2003). Enhancing learners' cognitive skills through multimedia design. Interactive Learning Environments, 11(1), 23-39. 
London, E. (1997). William Heard Kilpatrick. Revista trimestral de educación. (París. UNESCO: Oficina Internacional de Educación), XXVII (3), 503-521.Disponible en: http://www.ibe.unesco.org/sites/default/files/kilpatrs.PDF

Luy-Montejo, C. (2019, agosto). El aprendizaje basado en problemas (ABP) en el desarrollo de la inteligencia emocional de los estudiantes universitarios. Propósitos y Representaciones, 7(2), 353-383.

DOI: http://dx.doi.org/10.20511/pyr2019.v7n2.288.

Maldonado Pérez, M. (2008). Aprendizaje basado en proyectos colaborativos. Una experiencia en educación superior. Laurus, 14(28), 158-180. Disponible: https://www.redalyc.org/pdf/761/76111716009.pdf

Martins, V. F., Sampaio, P. N. M., Cordeiro, A. J. A., \& Viana, B. F. (2018). Implementing a Data Network Infrastructure Course using a Problem-based Learning Methodology. Journal of Information Systems Engineering and Management, 3(2), 10. https://doi.org/10.20897/jisem.201810.

May Cen, I. (2015). George Polya (1965). Cómo plantear y resolver problemas [título original: ¿How To Solve It?]. Diálogos en la Sociedad del Conocimiento, 3(8) 419-420. Recuperado de: https://www.redalyc.org/jatsRepo/4576/457644946012/html/index.html

Medina Rodríguez, P. (2017). La competencia Matemática a través de la resolución de problemas en educación secundaria [Tesis doctoral, Universidad de las Palmas de la Gran Canaria]. Disponible https://accedacris.ulpgc.es/bitstream/10553/26209/9/0745278_0000_0000.pdf

Michel, C., Lavoué, E., George, S. y Ji, M. (2018). Supporting Awareness and SelfRegulation in Project-Based Learning through Personalized Dashboards. International Journal of Technology Enhanced Learning, Vol. 9, pp.203-226. Recuperado de https://hal.archives-ouvertes.fr/hal-01384211.

DOI: 10.1504/IJTEL.2017.084500. hal-01384211

Ministerio de educación. (2017). Currículo Nacional de la educación básica. (Primera edición). Minedu. http://www.minedu.gob.pe/curriculo/pdf/curriculo-nacional2016.pdf 
Ministerio de educación. (2018). Guía docente para el aprovechamiento de las Tic. Minedu. Disponible en: http://www.perueduca.pe/recursosedu/c-librostexto/secundaria/ciencia-tecnologia/guia-tic-2019.pdf

Ministerio de educación. Oficina de medición de calidad de los aprendizajes (2016). Marco de fundamentación de las pruebas de la evaluación censal de estudiantes. Minedu-UMC. Disponible en http://umc.minedu.gob.pe/wpcontent/uploads/2016/04/Marco-de-Fundamentaci\%C3\%B3n-ECE.pdf

Ministerio de educación. (2012). Marco del Buen Desempeño Docente. Minedu. Disponible en: http://www.perueduca.pe/documents/60563/ce664fb7-a1dd-450da43d-bd8cd65b4736

Ministerio de educación. Oficina de medición de calidad de los aprendizajes. (2017). El Perú en Pisa 2015. Informe nacional de resultados. Minedu-UMC. Disponible en: http://umc.minedu.gob.pe/wp-content/uploads/2017/04/Libro_PISA.pdf

Ministerio de educación. Oficina de medición de calidad de los aprendizajes. (2019). Evaluación censal de estudiantes 2019: Informe de resultados para docentes. Minedu-UMC. Disponible en: http://umc.minedu.gob.pe/wpcontent/uploads/2020/06/Informe-para-docentes-de-Matematica-\%E2\%80\%932.\% $2 \%$ BA-grado-secundaria.pdf

Ministerio de educación. Oficina de medición de calidad de los aprendizajes. (2018). Evaluación PISA 2018. Minedu-UMC. Disponible en: http://umc.minedu.gob.pe/wp-content/uploads/2020/10/PPT-PISA2018_Web_vf-15-10-20.pdf

Ministerio de educación. (2013). Rutas de Aprendizaje: Los proyectos de aprendizaje para el logro de competencias. MINEDU. Disponible en: http://www.minedu.gob.pe/minedu/archivos/a/002/03-bibliografia-para-ebr/37proyecto.pdf

Ministerio de educación. (2015). Rutas de Aprendizaje versión 2015: ¿Qué y Cómo aprenden nuestros estudiantes? MINEDU.

Ministerio de educación. Sistema de consulta de resultados de evaluaciones. (2019). Evaluaciones nacionales de logros de aprendizaje 2019: ¿Qué aprendizajes logran nuestros estudiantes? Minedu-SICRECE. Disponible en: 
http://umc.minedu.gob.pe/wp-content/uploads/2020/06/Reporte-Nacional2019.pdf

Ministerio de educación. Sistema de consulta de resultados de evaluación. SICRECE. (2018). Informe de resultados para las instituciones educativas director/subdirector. Resultados de ECE: Un insumo para mejorar los aprendizajes. Minedu. SICRECE. Disponible https://drive.google.com/file/d/1eIDvSNEBP8OMULN3VV_nFQiYSmmUbq4q /view

Moldoveanu, M. y Narayandas, D. (15 de noviembre del 2019). The Future of Leadership Development. Harvard Bussines Review. pp. 1. Recuperado de https://hbr.org/2019/03/educating-the-next-generation-of-leaders

Neill, D. y Cortés Suarez, L. (2017). Procesos y fundamentos de la investigación científica. Editorial UTMACH. Disponible http://repositorio.utmachala.edu.ec/bitstream/48000/12498/1/Procesos-yFundamentosDeLainvestiagcionCientifica.pdf

Neira Churata, P. (2020). Influencia de la Aplicación del Método de Proyectos (MDP) en el Desarrollo de las Competencias Matemáticas en los Estudiantes del Servicio Nacional de Adiestramiento en el Trabajo Industrial (SENATI), 2019 [Tesis Para optar al Grado Académico de Doctor en Ciencias de la Educación, Universidad Nacional de Educación Enrique Guzmán y Valle]. Disponible en https://repositorio.une.edu.pe/handle/UNE/4748.

Nortes, A., Martínez R. (1994). Psicología Piagetiana y Educación Matemática. Revista interuniversitaria de formación del profesorado: RIFOP (21),59-70. Recuperado de: https://dialnet.unirioja.es/servlet/articulo?codigo $=117837$

Prieto García, A. y López Esteban, C. (2019, mayo). El efecto del aprendizaje Basado en Proyectos propio del BIE. Matemáticas, Educación y Sociedad, 2(1), 12-28. Disponible en: https://www.uco.es/ucopress/ojs/index.php/mes/article/view/12839/11657

Pujol Cunill, F. (2017). Aprendizaje basado en proyectos y el aprendizaje por descubrimiento guiado como estrategia didáctica en Biología y Geología de 4to de ESO [Trabajo fin de máster, Universidad Internacional de la Rioja]. Disponible en: 
https://reunir.unir.net/bitstream/handle/123456789/6052/PUJOL\%20CUNILL\% 2c\%20FRANCISCA.pdf?sequence $=1 \&$ isAllowed $=\mathrm{y}$

Qin, L. (2017). Design and Realization of Project-based Computer English Learning System. International Journal of Emerging Technologies in Learning, Vol. 12, No 08 (2017), pp.128-136. Recuperado de https://online-journals.org/index.php/ijet/article/view/7147/4558. DOI: https://doi.org/10.3991/ijet.v12.i08.7147

Rodríguez Jiménez, A. y Pérez Jacinto, A. (2017). Método científico de indagación y de construcción del conocimiento. Revista ean,82, p. 175-195. Disponible en: https://journal.universidadean.edu.co/index.php/Revista/article/view/1647/1661

Rodríguez Tortosa, M. (2018). ABP, Una propuesta para la Clase de Matemática [Tesis de fin de Master, Universidad de Almería]. http://repositorio.ual.es/bitstream/handle/10835/7129/TFM_RODRIGUEZ\%20T ORTOSA,\%20MARIA\%20JOSE.pdf?sequence $=1$

Sánchez Carlessi, H., Reyes Romero, C., Mejía Sáenz, K. (2018). Manual de términos en investigación científica, tecnológica y humanística. Universidad Ricardo Palma. Vicerrectorado de Investigación. https://www.urp.edu.pe/pdf/id/13350/n/libromanual-de-terminos-en-investigacion.pdf

Soler Rodríguez. G., Duardo Monteagudo, C. y Pulg Reyes, N. (2013). Factores educativos que inciden en el aprendizaje de los contenidos matemáticos en la universidad de las ciencias pedagógicas "Félix Valera Morales". Revista Iberoamericana sobre calidad, eficacia y cambio en educación, 11(4), 129-153. Disponible en: https://www.redalyc.org/articulo.oa?id=55128238008

Tantalean Salazar, H. (2020). Aprendizaje basado en problemas para desarrollar competencias matemáticas en estudiantes de primer grado del nivel secundario Trujillo 2019 [Tesis doctoral, Universidad César Vallejo]. https://repositorio.ucv.edu.pe/handle/20.500.12692/44492

Ursini S. (1996). Una perspectiva social para la educación matemática. Influencia de la teoría de L.S. Vygotsky. Educación Matemática, 08(03), 4249. http://funes.uniandes.edu.co/10063/

Useche M., Artigas W., Queipo B., Perozo E. (2020). Técnicas e instrumentos de recolección de datos cuali - cuantitativos. Universidad de la Guajira. 
https://www.researchgate.net/publication/344256464_Tecnicas_e_instrumentos de_recoleccion_de_datos_Cuali-Cuantitativos

Vara Horna, A. (2008). La tesis de maestría en educación: Una guía efectiva para obtener el grado de maestro y no desistir en el intento. Universidad de San Martín Porres. Disponible en: https://docplayer.es/12979423-Una-guia-efectiva-para-obtener-elgrado-de-maestro-y-no-desistir-en-el-intento.html

Vargas Vargas, N., Niño Vega, J. y Fernández Morales, F. (2020, marzo). Aprendizaje basado en proyectos mediados por TIC para superar dificultades en el aprendizaje de las operaciones básicas matemáticas. Redipe, 9(3),80-167. Disponible en: https://revista.redipe.org/index.php/1/article/view/943.

Vilca Paye, C. (2019, junio). Resolución de problemas como estrategia en el desarrollo de competencias matemáticas en estudiantes de secundaria. Revista de investigación de la Escuela de Posgrado: UNA Puno, 8(2),1028-1036. Disponible en: http://revistas.unap.edu.pe/epg/index.php/investigaciones/article/view/887

Wampash Antuash, D. (2018). Bajo rendimiento académico con los estudiantes del sexto $C$ de educación general básica de la Unidad didáctica tres de noviembre de la ciudad de Cuenca. [Tesis de Título de Licenciado en ciencias de educación, Universidad Politécnica Salesiana de la Cuenca]. Disponible en: https://dspace.ups.edu.ec/bitstream/123456789/16100/5/UPS-CT007793.pdf 